\title{
OCORRÊNCIA DE VERANICOS NO ESTADO DO RIO DE JANEIRO
}

\author{
ELEANDRO S. CRUZ ${ }^{1}$, DANIEL F. CARVALHO ${ }^{2}$, MARCOS B. CEDDIA ${ }^{3}$, \\ MAURO A. H. ANTUNES ${ }^{4}$, RENATO M. AQUINO
}

\begin{abstract}
RESUMO: O objetivo deste trabalho foi simular veranicos com diferentes durações e identificar suas respectivas probabilidades de ocorrência para dezembro, janeiro e fevereiro no Estado do Rio de Janeiro. Para isso, foram utilizadas séries históricas pluviométricas, com período base de 16 anos referentes a 40 estações localizadas no Estado do Rio de Janeiro, 15 em Minas Gerais, 4 no Espírito Santo e 3 em São Paulo. Com os resultados obtidos, pôde-se concluir que, em geral, as maiores probabilidades de ocorrência de veranicos são previstas para o mês de dezembro, diminuindo para janeiro e, em seguida, para fevereiro. Os veranicos com durações de 1-5 dias e 6-10 dias ocorreram com maior probabilidade no Estado. A maior probabilidade de ocorrência de veranicos com 11-15 dias de duração ocorreu na região Norte do Estado, sobretudo no mês de fevereiro. A região Sul apresentou maior probabilidade de ocorrências de veranicos de 1-5 dias de duração.
\end{abstract}

PALAVRAS-CHAVE: dias secos, simulação.

\section{OCURRENCE OF DROUGHTS IN RIO DE JANEIRO STATE, BRAZIL}

SUMMARY: The objective of this work was to simulate droughts with different duration ranges (1-5, 6-10 and 11-15 days) in order to identify its occurrence probability in Rio de Janeiro State, Brazil, between December to February. Rain gauge data collected over 16 years from 62 weather stations, 40 from Rio de Janeiro, 15 from Minas Gerais, 4 from Espírito Santo and 3 from São Paulo, were used in the analysis. The results allowed the conclusion that, in general, the highest drought probabilities were found for December, decreasing in January and from January to February. Drought periods lasting 1-5 and 6-10 days were found to occur with greater probability in the State. The probability of droughts lasting 11-15 days was higher in Northern region, particularly in February. The Southern State region presented higher probability of droughts for periods of 1-5 days.

KEYWORDS: rainfall shortage, dry days, simulation.

\section{INTRODUÇÃO}

No Brasil, existem 10 milhões de hectares utilizados para agricultura, sendo aproximadamente 3,2 milhões irrigados. Do total irrigado, apenas 36 mil hectares estão no Estado do Rio de Janeiro, que apresenta valores de área irrigada muito inferiores aos demais estados da região Sudeste, (CRISTHOFIDIS, 2003). Embora não seja um dos mais tradicionais produtores agrícolas do Brasil, o Rio de Janeiro vem apresentando um avanço na produção agrícola irrigada, destacando-se o pólo de fruticultura, localizado nas regiões Norte e Noroeste do Estado.

\footnotetext{
${ }_{1}^{1}$ Estudante de Agronomia da UFRRJ, Bolsista de iniciação científica PIBIC/UFRRJ/CNPq.

${ }^{2}$ Professor Adjunto, DE/IT/UFRRJ, Seropédica - RJ, Fone: (0XX21) 2682.1865, e-mail: carvalho@ufrrj.br. Bolsista do CNPq.

${ }^{3}$ Professor Adjunto, DS/IA/UFRRJ, Seropédica - RJ, e-mail: ceddia@ufrrj.br.

${ }^{4}$ Professor Adjunto, DE/IT/UFRRJ, Seropédica - RJ, e-mail: mantunes@ufrrj.br.

${ }^{5}$ Estudante de Agronomia da UFRRJ.

Recebido pelo Conselho Editorial em: 12-9-2002

Aprovado pelo Conselho Editorial em: 2-2-2004
} 
Na maior parte das terras agricultáveis, não se utiliza a irrigação, dependendo-se exclusivamente dos regimes pluviais. Em algumas regiões, principalmente nos cerrados, a precipitação total do período chuvoso é suficiente para o desenvolvimento da agricultura, porém é comum a ocorrência de seqüência de dias secos durante a estação chuvosa, o que é conhecido como veranico (SOUSA, 1999). Esse fato é também verificado em algumas regiões do Estado do Rio de Janeiro, principalmente aquelas que se destacam na produção agrícola. Assim, para os planejadores de sistemas de produção, é importante conhecer o comportamento dos elementos climáticos em cada localidade, especialmente do regime pluviométrico, para subsidiá-los na tarefa de otimização do aproveitamento dos recursos naturais e na obtenção da máxima produção ao menor custo possível (PAIVA, 1997).

Um erro no planejamento ou o desconhecimento do regime de chuva na produção de sequeiro, pode acarretar um déficit hídrico nas plantas, que se caracteriza por uma redução no seu conteúdo de água e do seu potencial hídrico. A ocorrência desses fatos pode levar ao fechamento estomático, perda de turgidez celular e redução do crescimento das plantas, tendo como conseqüência a redução da produção final e prejuízo econômico.

Com a necessidade de aumentar a produção brasileira, quanto mais informações estiverem disponíveis a respeito das variáveis climáticas, melhor será a orientação dada aos produtores para a tomada de decisão a respeito da época de plantio e/ou dimensionamento e manejo de sistemas irrigados. Vários estudos mostram que a definição das épocas de plantio por meio da análise da quantidade e distribuição pluvial pode contribuir para diminuir o risco de deficiência hídrica das culturas, como revela o trabalho de ASSAD et al. (1998). Um tratamento qualitativo do veranico é fundamental a fim de identificar o seu comportamento espaço-temporal nas regiões mais e menos afetadas, além de um tratamento quantitativo determinando-se a amplitude, freqüência e a probabilidade de ocorrência (PAIVA, 1997). Segundo HASHEMI \& DECKER (1969), conhecendo-se a duração dos períodos secos, a irrigação pode ser aplicada de forma a satisfazer as exigências do período, e uma economia de água pode ser conseguida.

Para a determinação da duração de um período seco, é necessário o estabelecimento de um valorlimite de precipitação, abaixo do qual um dia pode ser considerado como seco. Por outro lado, dias chuvosos são aqueles nos quais a precipitação supera o limite preestabelecido. Para a determinação dos períodos secos de diferentes durações, devem ser observadas todas as ocorrências de seqüências de dias secos intercalados entre dias chuvosos (SOUSA, 1999). Existem vários critérios utilizados para a determinação de dia seco e dia chuvoso. O critério adotado por SILVA et al. (1977) pode ser considerado mais coerente e consiste em denominar como dia seco aquele no qual a precipitação diária for inferior à evapotranspiração média diária da região de estudo. Outros autores consideram como dia seco a precipitação inferior ao dia que teve o menor registro.

Tendo em vista a importância do estudo da ocorrência de períodos secos em regiões agrícolas, desenvolveu-se este trabalho com o objetivo de estimar as freqüências de ocorrência de veranicos de diferentes durações no Estado do Rio de Janeiro, situado na região Sudeste do Brasil.

\section{MATERIAL E MÉTODOS}

Este trabalho foi realizado para o Estado do Rio de Janeiro, considerando os meses de dezembro, janeiro e fevereiro, que se caracterizam pelos maiores índices pluviométricos na região. Foram utilizados dados pluviométricos obtidos da Agência Nacional de Energia Elétrica (ANEEL). Para manter a uniformidade dos dados, foram utilizadas séries históricas com período-base de 16 anos (1982-1997). Com base nesse período, foram selecionadas 40 estações localizadas no Estado do Rio de Janeiro, quatro no Espírito Santo, 15 em Minas Gerais e três em São Paulo. As estações pertencentes aos três últimos Estados serviram de apoio para a melhoria da interpolação dos dados. Na Figura 1, é apresentado o mapa-base do Estado do Rio de Janeiro com as estações pluviométricas selecionadas. 
De acordo com SOUSA (1999), foi considerado como dia seco aquele em que a lâmina precipitada diária era inferior à lâmina média de evapotranspiração de referência (ETo) do mês considerado. Por isso, foram utilizados os dados de CARVALHO et al. (2000), que estimaram a ETo para o Estado do Rio de Janeiro utilizando o método de Penman-Monteith. Na Figura 2, apresentam-se os valores médios de ETo para o Estado em dezembro, janeiro e fevereiro. Em função da localização geográfica de cada posto pluviométrico, considerou-se como limite de ocorrência do veranico, a lâmina média de cada classe de ETo. Na Tabela 1, apresenta-se a listagem com a localização geográfica de todas as estações e os respectivos valores de ETo para os meses considerados.

De posse dos arquivos pluviométricos e do valor mínimo de chuva (ETo) considerado para cada localidade, procedeu-se à pesquisa do número de veranicos para cada duração, nos referidos meses. Para isso, foi desenvolvido, em linguagem DELPHI 5.0, um programa computacional que rapidamente permitiu a obtenção dos resultados, gerando arquivos em um formato adequado para estimar a ocorrência do veranico, que foi realizada utilizando o programa computacional VERANICO, desenvolvido por SOUSA (1999). Esse programa simula mensalmente o veranico, ou seja, são efetuadas simulações das freqüências relativas e dos períodos de retorno para cada veranico de diferentes durações, ocorridos em um mês predeterminado pelo usuário. Essa simulação baseia-se na distribuição de probabilidade empírica, na qual são gerados números aleatórios que são comparados com a freqüência acumulada e, a partir dessa, são determinadas as diferentes freqüências de durações dos veranicos. Com base nessa distribuição, SOUSA (1999) desenvolveu esse programa, utilizando a metodologia Monte Carlo, a qual utiliza a comparação de números randômicos com uma determinada função estatística (NEUMANN \& ULAM, 1949; SOBOL, 1983).

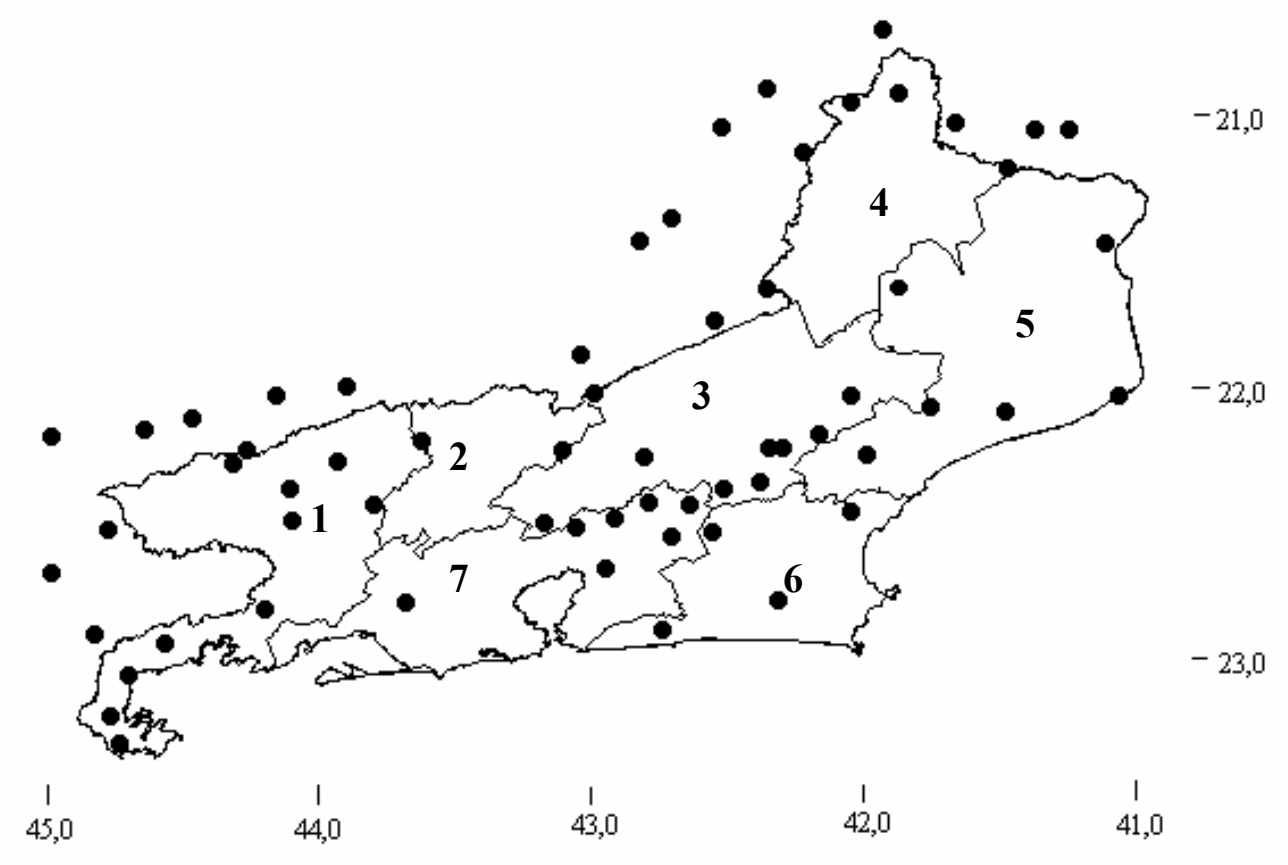

FIGURA 1. Mapa-base do Estado do Rio de Janeiro com as estações pluviométricas selecionadas e mesorregiões (1-Sul; 2 - Centro-Sul; 3 - Serrana; 4 - Noroeste; 5 - Norte; 6 - Litorânea; 7 - Metropolitana). 


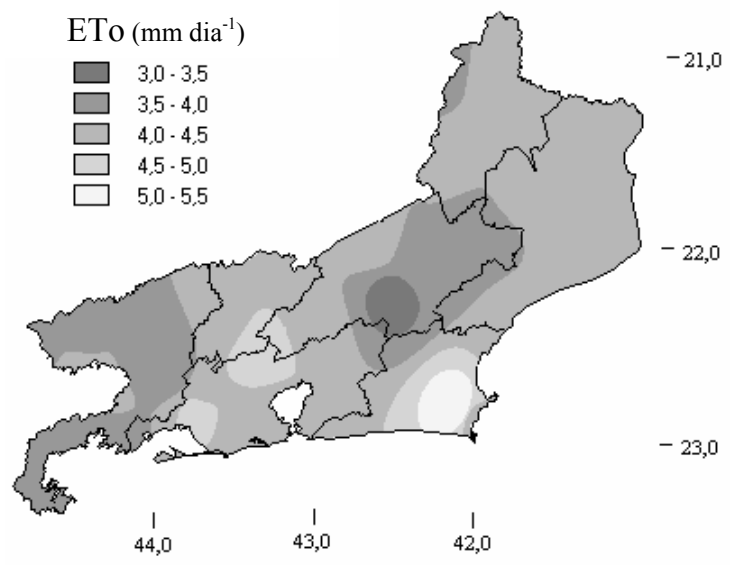

(a)

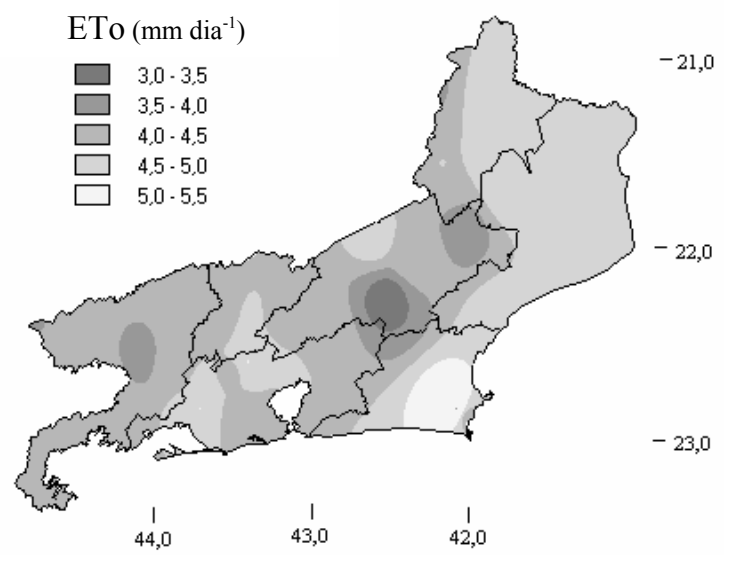

(b)

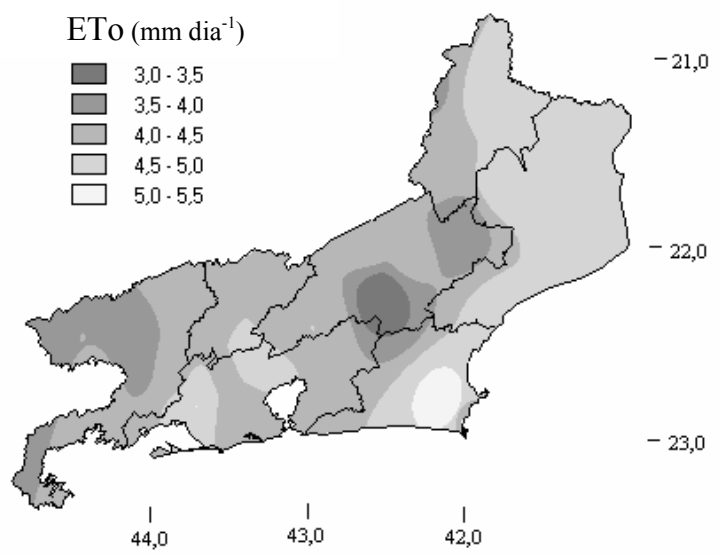

(c)

|FIGURA 2. Mapas dos valores de ETo no Estado do Rio de Janeiro para dezembro (a), janeiro (b) e fevereiro (c). Fonte: CARVALHO et al. (2000).

TABELA 1. Descrição das estações pluviométricas utilizadas no trabalho e valores de evapotranspiração de referência (ETo).

\begin{tabular}{|c|c|c|c|c|c|c|c|}
\hline \multicolumn{8}{|c|}{ Rio de Janeiro } \\
\hline \multirow{2}{*}{ Código } & \multirow{2}{*}{ Estação } & \multirow{2}{*}{ Local } & \multirow{2}{*}{ Latitude } & \multirow{2}{*}{ Longitude } & \multicolumn{3}{|c|}{ Eto $\left(\mathrm{mm} \mathrm{dia}^{-1}\right)$} \\
\hline & & & & & dez. & jan. & fev. \\
\hline 2041046 & Varre-Sai & Natividade & $20^{\circ} 55^{\prime} 51^{\prime \prime}$ & $41^{\circ} 51^{\prime} 51^{\prime \prime}$ & 4,25 & 4,75 & 4,75 \\
\hline 2241001 & Farol S. Tomé & Campos & $22^{\circ} 02^{\prime} 35^{\prime \prime}$ & $41^{\circ} 03^{\prime} 03^{\prime \prime}$ & 4,25 & 4,75 & 4,75 \\
\hline 2241002 & Usina Quissamã & Macaé & $22^{\circ} 06^{\prime} 25^{\prime \prime}$ & $41^{\circ} 28^{\prime} 28^{\prime \prime}$ & 4,25 & 4,75 & 4,75 \\
\hline 2241003 & Macabuzinho & Conceição Macabu & $22^{\circ} 05^{\prime} 11^{\prime \prime}$ & $41^{\circ} 44^{\prime} 44^{\prime \prime}$ & 4,25 & 4,75 & 4,25 \\
\hline 2241004 & Faz. Oratori & Macaé & $22^{\circ} 15^{\prime} 34^{\prime \prime}$ & $41^{\circ} 58^{\prime} 58^{\prime \prime}$ & 4,25 & 4,75 & 4,75 \\
\hline 2242001 & Leitão da Cunha & Trajano de Moraes & $22^{\circ} 02^{\prime} 34^{\prime \prime}$ & $42^{\circ} 02^{\prime} 02^{\prime \prime}$ & 3,75 & 3,75 & 3,75 \\
\hline 2242002 & Maria Mendonça & Trajano de Moraes & $22^{\circ} 11^{\prime} 11^{\prime \prime}$ & $42^{\circ} 09^{\prime} 09^{\prime \prime}$ & 3,75 & 4,25 & 4,25 \\
\hline 2242003 & Piller & Nova Friburgo & $22^{\circ} 14^{\prime} 17^{\prime \prime}$ & $42^{\circ} 20^{\prime} 20^{\prime \prime}$ & 3,75 & 4,25 & 3,75 \\
\hline 2242004 & Galdinópolis & Nova Friburgo & $22^{\circ} 21^{\prime} 49^{\prime \prime}$ & $42^{\circ} 22^{\prime} 22^{\prime \prime}$ & 3,75 & 3,75 & 3,75 \\
\hline 2242005 & Faz. São João & Nova Friburgo & $22^{\circ} 23^{\prime} 22^{\prime \prime}$ & $42^{\circ} 30^{\prime} 30^{\prime \prime}$ & 3,75 & 3,75 & 3,75 \\
\hline 2242006 & Rio Dourado & Casimiro de Abreu & $22^{\circ} 28^{\prime} 24^{\prime \prime}$ & $42^{\circ} 02^{\prime} 05^{\prime \prime}$ & 4,25 & 4,75 & 4,75 \\
\hline 2242007 & Quartéis & Silva Jardim & $22^{\circ} 47^{\prime} 44^{\prime \prime}$ & $42^{\circ} 18^{\prime} 18^{\prime \prime}$ & 4,25 & 4,75 & 4,75 \\
\hline 2242008 & Gaviões & Silva Jardim & $22^{\circ} 32^{\prime} 56^{\prime \prime}$ & $42^{\circ} 32^{\prime} 32^{\prime \prime}$ & 3,75 & 3,75 & 3,75 \\
\hline 2242010 & anuel Ribei & Maricá & $22^{\circ} 54^{\prime} 24^{\prime \prime}$ & $42^{\circ} 43^{\prime} 43^{\prime \prime}$ & 4,25 & 4,75 & 4,25 \\
\hline 2242011 & Estação de Bomba & Magé & $22^{\circ} 40^{\prime} 49^{\prime \prime}$ & $42^{\circ} 56^{\prime} 56^{\prime \prime}$ & 3,75 & 4,25 & 4,25 \\
\hline 2242012 & Represa do Pará & Magé & $22^{\circ} 29^{\prime} 55^{\prime \prime}$ & $42^{\circ} 54^{\prime} 54^{\prime \prime}$ & 4,25 & 4,25 & 4,25 \\
\hline 2242013 & Faz. do Carmo & Cacho. de Macacu & $22^{\circ} 26^{\prime} 17^{\prime \prime}$ & $42^{\circ} 46^{\prime} 46^{\prime \prime}$ & 3,75 & 4,25 & 4,25 \\
\hline
\end{tabular}




\begin{tabular}{|c|c|c|c|c|c|c|c|}
\hline 2242014 & Japuiba & Cacho. de Macacu & $22^{\circ} 33^{\prime} 33^{\prime \prime}$ & $42^{\circ} 41^{\prime} 41^{\prime \prime}$ & 3,75 & 4,25 & 4,25 \\
\hline 2242016 & Faz. S. João & Cacho. de Macacu & $22^{\circ} 26^{\prime} 58^{\prime \prime}$ & $42^{\circ} 37^{\prime} 37^{\prime \prime}$ & 3,75 & 4,25 & 4,25 \\
\hline 2243001 & Andorinhas & Magé & $22^{\circ} 32^{\prime} 00^{\prime \prime}$ & $43^{\circ} 03^{\prime} 03^{\prime \prime}$ & 4,25 & 4,25 & 4,25 \\
\hline 2244028 & Faz. Fortaleza & Angra dos Reis & $22^{\circ} 57^{\prime} 30^{\prime \prime}$ & $44^{\circ} 33^{\prime} 33^{\prime \prime}$ & 4,25 & 4,25 & 4,25 \\
\hline 2344006 & Patrimônio & Parati & $23^{\circ} 19^{\prime} 03^{\prime \prime}$ & $44^{\circ} 43^{\prime} 43^{\prime \prime}$ & 3,75 & 4,25 & 3,75 \\
\hline 2344007 & Parati & Parati & $23^{\circ} 13^{\prime} 25^{\prime \prime}$ & $44^{\circ} 45^{\prime} 45^{\prime \prime}$ & 3,75 & 4,25 & 3,75 \\
\hline 2344008 & São Roque & Parati & $23^{\circ} 04^{\prime} 20^{\prime \prime}$ & $44^{\circ} 41^{\prime} 41^{\prime \prime}$ & 3,75 & 4,25 & 3,75 \\
\hline 2042027 & Porciúncula & Porciúncula & $20^{\circ} 57^{\prime} 48^{\prime \prime}$ & $42^{\circ} 02^{\prime} 14^{\prime \prime}$ & 4,25 & 4,25 & 4,25 \\
\hline 2141001 & São Francisco P. & São João da Barra & $21^{\circ} 28^{\prime} 58^{\prime \prime}$ & $41^{\circ} 06^{\prime} 12^{\prime \prime}$ & 4,25 & 4,75 & 4,75 \\
\hline 2141006 & Dois Rios & São Fidélis & $21^{\circ} 38^{\prime} 36^{\prime \prime}$ & $41^{\circ} 51^{\prime} 31^{\prime \prime}$ & 4,25 & 4,75 & 4,75 \\
\hline 2242018 & Barra Alegre & Bom Jardim & $22^{\circ} 14^{\prime} 08^{\prime \prime}$ & $42^{\circ} 17^{\prime} 09^{\prime \prime}$ & 3,75 & 4,25 & 4,25 \\
\hline 2242026 & Bom Sucesso & Teresópolis & $22^{\circ} 16^{\prime} 17^{\prime \prime}$ & $42^{\circ} 47^{\prime} 41^{\prime \prime}$ & 3,25 & 3,25 & 3,25 \\
\hline 2242028 & Anta & Sapucaia & $22^{\circ} 02^{\prime} 07^{\prime \prime}$ & $42^{\circ} 59^{\prime} 27^{\prime \prime}$ & 4,25 & 4,25 & 4,25 \\
\hline 2243002 & Barra do Piraí & Barra do Piraí & $22^{\circ} 26^{\prime} 58^{\prime \prime}$ & $43^{\circ} 47^{\prime} 56^{\prime \prime}$ & 4,25 & 4,25 & 4,25 \\
\hline 2243004 & Conservatória & Valença & $22^{\circ} 17^{\prime} 15^{\prime \prime}$ & $43^{\circ} 55^{\prime} 46^{\prime \prime}$ & 4,25 & 4,25 & 4,25 \\
\hline 2243007 & Taboas & Rio das Flores & $22^{\circ} 12^{\prime} 31^{\prime \prime}$ & $43^{\circ} 37^{\prime} 24^{\prime \prime}$ & 4,25 & 4,25 & 4,25 \\
\hline 2243009 & Petrópolis & Petrópolis & $22^{\circ} 30^{\prime} 42^{\prime \prime}$ & $43^{\circ} 10^{\prime} 15^{\prime \prime}$ & 4,25 & 4,25 & 4,25 \\
\hline 2243013 & Areal & Três Rios & $22^{\circ} 14^{\prime} 31^{\prime \prime}$ & $43^{\circ} 06^{\prime} 16^{\prime \prime}$ & 4,25 & 4,25 & 4,25 \\
\hline 2244037 & Fumaça & Resende & $22^{\circ} 17^{\prime} 51^{\prime \prime}$ & $44^{\circ} 18^{\prime} 38^{\prime \prime}$ & 3,75 & 4,25 & 3,75 \\
\hline 2244040 & Lidice & Rio Claro & $22^{\circ} 39^{\prime} 56^{\prime \prime}$ & $44^{\circ} 11^{\prime} 56^{\prime \prime}$ & 3,75 & 3,75 & 3,75 \\
\hline 2244041 & Volta Redonda & Volta Redonda & $22^{\circ} 30^{\prime} 04^{\prime \prime}$ & $44^{\circ} 05^{\prime} 31^{\prime \prime}$ & 3,75 & 3,75 & 3,75 \\
\hline 2244045 & Nossa Senhora & Barra Mansa & $22^{\circ} 23^{\prime} 08^{\prime \prime}$ & $44^{\circ} 06^{\prime} 27^{\prime \prime}$ & 3,75 & 4,25 & 3,75 \\
\hline \multicolumn{8}{|c|}{ Espírito Santo } \\
\hline 2141014 & Pte. de Itabapoana & Mimoso do Sul & $21^{\circ} 12^{\prime} 22^{\prime \prime}$ & $41^{\circ} 27^{\prime} 46^{\prime \prime}$ & 4,25 & 4,75 & 4,75 \\
\hline 2141015 & Mimoso do Sul & Mimoso do Sul & $21^{\circ} 03^{\prime} 53^{\prime \prime}$ & $41^{\circ} 21^{\prime} 45^{\prime \prime}$ & 4,25 & 4,75 & 4,75 \\
\hline 2141016 & S. José do Calçado & São José do Cal & $21^{\circ} 02^{\prime} 12^{\prime \prime}$ & $41^{\circ} 39^{\prime} 08^{\prime \prime}$ & 4,25 & 4,75 & 4,75 \\
\hline 2141017 & S. José das Torres & Mimoso do Sul & $21^{\circ} 03^{\prime} 45^{\prime \prime}$ & $41^{\circ} 14^{\prime} 28^{\prime \prime}$ & 4,25 & 4,75 & 4,75 \\
\hline 2041005 & Caiana & Caiana & $20^{\circ} 41^{\prime} 38^{\prime \prime}$ & $41^{\circ} 55^{\prime} 19^{\prime \prime}$ & 4,25 & 4,75 & 4,75 \\
\hline 2244057 & Ponte do Costa & Bocaina de Minas & $22^{\circ} 07^{\prime} 41^{\prime \prime}$ & $44^{\circ} 27^{\prime} 31^{\prime \prime}$ & 3,75 & 3,75 & 3,75 \\
\hline 2244064 & Pedreira & Bom Jardim & $22^{\circ} 02^{\prime} 33^{\prime \prime}$ & $44^{\circ} 09^{\prime} 12^{\prime \prime}$ & 3,75 & 3,75 & 3,75 \\
\hline 2244065 & Alagoa & Alagoa & $22^{\circ} 10^{\prime} 13^{\prime \prime}$ & $44^{\circ} 38^{\prime} 12^{\prime \prime}$ & 3,75 & 3,75 & 3,75 \\
\hline 2244071 & Pouso Alto & Pouso Alto & $22^{\circ} 11^{\prime} 36^{\prime \prime}$ & $44^{\circ} 58^{\prime} 30^{\prime \prime}$ & 3,75 & 4,25 & 3,75 \\
\hline 2142001 & Cataguazes & Cataguazes & $21^{\circ} 23^{\prime} 22^{\prime \prime}$ & $42^{\circ} 41^{\prime} 47^{\prime \prime}$ & 3,75 & 4,25 & 3,75 \\
\hline 2243002 & Faz. São Gabriel & Rio Preto & $22^{\circ} 00^{\prime} 42^{\prime \prime}$ & $43^{\circ} 53^{\prime} 30^{\prime \prime}$ & 3,75 & 4,25 & 3,75 \\
\hline 2244036 & Zelinda & Passa Vinte & $22^{\circ} 14^{\prime} 35^{\prime \prime}$ & $44^{\circ} 15^{\prime} 49^{\prime \prime}$ & 3,75 & 4,25 & 3,75 \\
\hline 2142002 & Patr. do Muriaé & Patr. do Muriaé & $21^{\circ} 08^{\prime} 55^{\prime \prime}$ & $42^{\circ} 12^{\prime} 56^{\prime \prime}$ & 3,75 & 4,25 & 3,75 \\
\hline 2142004 & Muriaé & Muriaé & $21^{\circ} 03^{\prime} 01^{\prime \prime}$ & $42^{\circ} 30^{\prime} 56^{\prime \prime}$ & 3,75 & 4,25 & 3,75 \\
\hline 2142006 & Usina Maurício & Itamarati de Minas & $21^{\circ} 28^{\prime} 17^{\prime \prime}$ & $42^{\circ} 48^{\prime} 45^{\prime \prime}$ & 3,75 & 4,25 & 3,75 \\
\hline 2142007 & Faz da Barra & Pirapetinga & $21^{\circ} 39^{\prime} 29^{\prime \prime}$ & $42^{\circ} 20^{\prime} 34^{\prime \prime}$ & 3,75 & 4,25 & 3,75 \\
\hline 2142008 & Volta Grande & Volta Grande & $21^{\circ} 46^{\prime} 09^{\prime \prime}$ & $42^{\circ} 32^{\prime} 23^{\prime \prime}$ & 3,75 & 4,25 & 3,75 \\
\hline 2142009 & Jussara & Miradouro & $20^{\circ} 54^{\prime} 41^{\prime \prime}$ & $42^{\circ} 20^{\prime} 58^{\prime \prime}$ & 3,75 & 4,25 & 3,75 \\
\hline 2143013 & Estêvão Pinto & Mar de Espanha & $21^{\circ} 53^{\prime} 47^{\prime \prime}$ & $43^{\circ} 02^{\prime} 29^{\prime \prime}$ & 3,75 & 4,25 & 3,75 \\
\hline \multicolumn{8}{|c|}{ São Paulo } \\
\hline 2244001 & Queluz & Queluz & $22^{\circ} 32^{\prime} 25^{\prime \prime}$ & $44^{\circ} 46^{\prime} 23^{\prime \prime}$ & 3,75 & 4,25 & 3,75 \\
\hline 2244010 & Bocaina & Cachoeira Paulista & $22^{\circ} 41^{\prime} 31^{\prime \prime}$ & $44^{\circ} 58^{\prime} 30^{\prime \prime}$ & 3,75 & 4,25 & 3,75 \\
\hline 2244048 & Cunha & Cunha & $22^{\circ} 55^{\prime} 16^{\prime \prime}$ & $44^{\circ} 49^{\prime} 20^{\prime \prime}$ & 3,75 & 4,25 & 3,75 \\
\hline
\end{tabular}

Com os dados de freqüência de ocorrência de veranicos obtidos para uma dada duração, foram calculadas, para cada localidade, as probabilidades de ocorrência dos mesmos com duração de até cinco dias, de 6-10 dias e de 11-15 dias, conforme eq.(1). Nessa equação, Na foi igual a 16 e a FRSi foi obtida somando-se as freqüências de ocorrências de veranico nas durações citadas anteriormente. A probabilidade de ocorrência foi obtida tomando-se o inverso do período de retorno, para cada intervalo considerado.

$$
\mathrm{PRS}_{\mathrm{i}}=\frac{\mathrm{Na}}{\mathrm{FRS}_{\mathrm{i}} \mathrm{FTO}}
$$

em que, 
$\mathrm{PRS}_{\mathrm{i}}$ - período de retorno simulado para um veranico de duração correspondente a i dias;

$\mathrm{Na}$ - número de anos da série utilizada;

$\mathrm{FRS}_{\mathrm{i}}$ - freqüência relativa simulada dos veranicos de i dias, e

FTO - freqüência total observada.

Foi determinado, também, o valor do veranico médio ponderado, o qual foi obtido pela multiplicação da duração de cada veranico pela respectiva freqüência simulada, dividido pela soma total das freqüências.

As coordenadas geográficas de cada estação (Tabela 1) foram usadas para criar arquivos georreferenciados contendo as probabilidades de ocorrência de veranicos com períodos de 1-5 dias, 610 dias e 11-15 dias, e as durações médias ponderadas de cada veranico, para os meses estudados. A partir desses arquivos, o programa IDRISI 3.2 foi utilizado para a execução das seguintes etapas:

- Interpolação dos dados pontuais, utilizando o módulo INTERPOL, obtendo-se imagens digitais com 1.016 colunas e 712 linhas, o que correspondeu a um tamanho de célula de, aproximadamente, $500 \mathrm{~m}$ x $500 \mathrm{~m}$;

- Reclassificação das imagens com o módulo RECLASS, a fim de obter mapas com classes em intervalos de $0,5 \%$ de probabilidade e intervalos diários de veranicos;

- Utilização do módulo OVERLAY, em que o mapa-base do Estado do Rio de Janeiro foi multiplicado pelos mapas gerados anteriormente, obtendo-se os mapas temáticos finais, e temática.

- Utilização do módulo AREA, para a determinação da área do Estado ocupada com cada classe

O método de interpolação utilizado neste estudo foi o do inverso da distância ao quadrado (IQD). Resultados obtidos por VALERIANO et al. (2002), em seus estudos para o Estado de São Paulo, revelaram que a supressão da anisotropia, que pode ser compreendida como a diferença no comportamento dos dados em diferentes direções, ao utilizar método de interpolação IQD, causou o aparecimento de numerosas formas concêntricas em torno dos postos de coleta, com um fundo médio de valor relativamente constante em torno desses; esses autores constataram, ainda, que a incorporação da anisotropia no método de interpolação resultou em contornos de classes harmoniosos com a topografia, o que contribuiu para a redução da coalescência dos valores de postos de observação que, embora próximos, pertençam a áreas geomorfológica e climaticamente distintas. Neste estudo, não foram levados em consideração os efeitos da variabilidade espacial/anisotropia sobre a probabilidade de ocorrência dos veranicos no Estado, porém, dentre os métodos de interpolação que não levam em consideração a dependência espacial das variáveis disponíveis, esse consiste no que melhor se adapta à função.

\section{RESULTADOS E DISCUSSÃO}

\section{Ocorrência de veranicos para os diferentes meses}

Após a análise dos resultados obtidos pelo programa de simulação do veranico, foi possível ter uma noção da ocorrência do fenômeno no Estado. Na Tabela 2, apresentam-se os maiores e os menores valores de probabilidade simulados para cada duração e meses estudados. Pode-se perceber que, em geral, as maiores probabilidades de ocorrência de veranicos ocorrem no mês de dezembro, diminuindo para janeiro, e em seguida para fevereiro, exceto para os veranicos com duração de 11 a 15 dias. Para essa duração, as probabilidades de ocorrência são baixas para os três meses, sendo maior em fevereiro.

TABELA 2. Valores extremos de probabilidade de ocorrência de veranico simulados para o Estado do Rio de Janeiro, em porcentagem, com durações de 1-5 dias, 6-10 dias e 11-15 dias. 


\begin{tabular}{|c|c|c|c|c|c|c|c|c|c|}
\hline \multirow{2}{*}{ Valores } & \multicolumn{3}{|c|}{ dezembro } & \multicolumn{3}{|c|}{ janeiro } & \multicolumn{3}{|c|}{ fevereiro } \\
\hline & 1 a 5 & 6 a 10 & 11 a 15 & 1 a 5 & 6 a 10 & 11 a 15 & 1 a 5 & 6 a 10 & 11 a 15 \\
\hline$\overline{\text { Míni }}$ & 1,93 & 0,27 & 0,0 & 1,89 & 0,29 & 0,0 & 1,0 & 0,27 & 0,05 \\
\hline Máximo & 6,27 & 1,34 & 0,46 & 6,27 & 1,17 & 0,41 & 4,88 & 1,14 & 0,58 \\
\hline
\end{tabular}

As localizações geográficas das áreas de maior e/ou menor probabilidade de ocorrências podem ser observadas nas Figuras 3, 4 e 5, para os meses de dezembro, janeiro e fevereiro, respectivamente. Com base na Tabela 2, percebe-se, na Figura 3, que não está sendo apresentado o mapa referente ao período de 11 a 15 dias, uma vez que os valores extremos de probabilidade de ocorrências variam de 0,0 a 0,46; portanto, toda a área do Estado ficou em uma mesma classe. O mesmo fato é observado para janeiro (Figura 4). Ainda com o auxílio da Tabela 2, observa-se, na Figura 3a, a ocorrência de dez classes de probabilidade de ocorrência de veranicos com duração de 1 a 5 dias, variando de 1,5 a 2,0\% até 6,0 a $6,5 \%$. Pode-se observar, também, que a distribuição das classes está bastante variada em toda a área do Estado, sendo observada a maior probabilidade de ocorrência na região Sul, limite com Minas Gerais e São Paulo, e as menores, nas regiões Centro-Sul e Norte do Estado.

Em comparação com a Figura 3b (duração de 6-10 dias), fica claro que, em geral, regiões que apresentam baixa probabilidade de ocorrência de veranicos de curta duração são aquelas em que maiores probabilidades são esperadas para veranicos de 6-10 dias. Para essa duração, a região Noroeste do Estado apresenta a maior probabilidade de ocorrência, estando na faixa de 1,0 a 1,5\% de probabilidade.

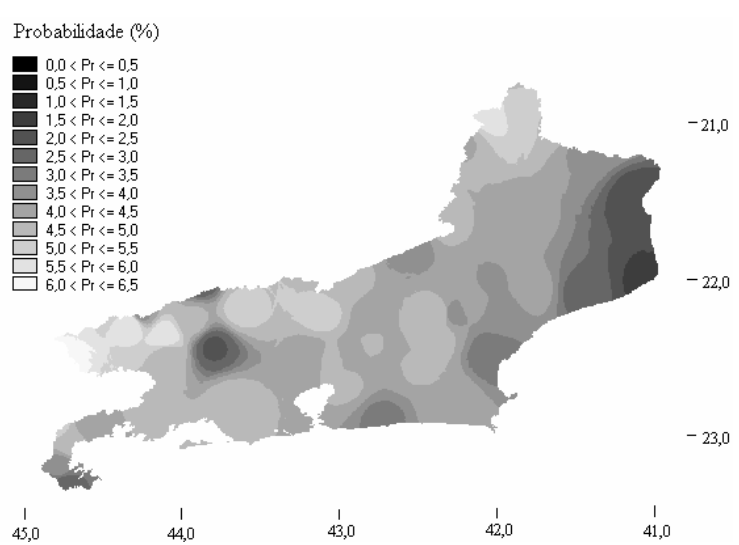

(a)

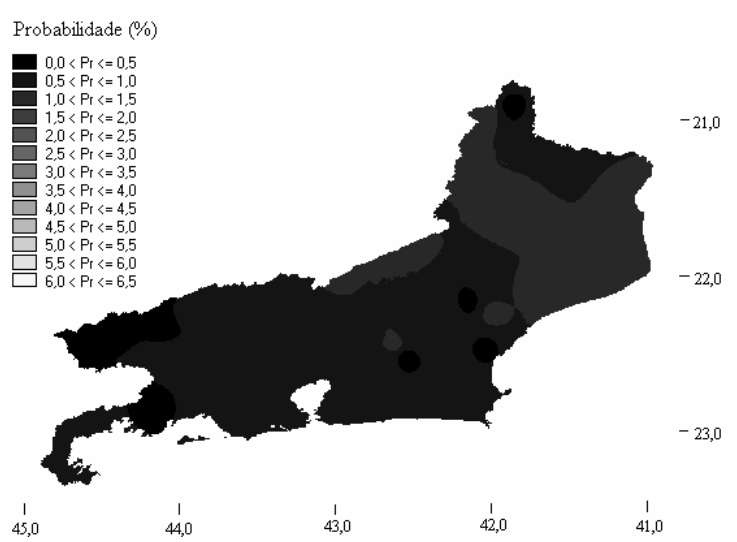

(b)

FIGURA 3. Mapas dos valores de probabilidade de veranico no Estado do Rio de Janeiro com duração de 1-5 dias (a), 6-10 dias (b), no mês de dezembro.

Na Figura 4a, pode-se observar que, no mês de dezembro, a região Norte do Estado é a que apresenta menores probabilidades de ocorrência de veranicos de 1-5 dias de duração, enquanto as maiores probabilidades de ocorrência limitam-se à região Sul, parte do Centro-Sul e da região Noroeste. Para os veranicos com duração de 6-10 dias (Figura 4b), observa-se que as probabilidades de ocorrência são baixas em todo o Estado, sendo as maiores probabilidades observadas em pequenas manchas restritas ao Centro-Sul, ocupando uma área muito pequena em relação ao restante do Estado. 


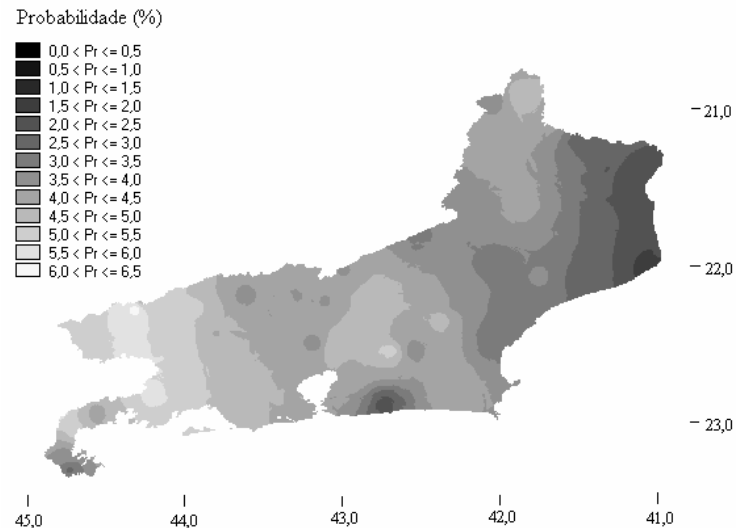

(a)

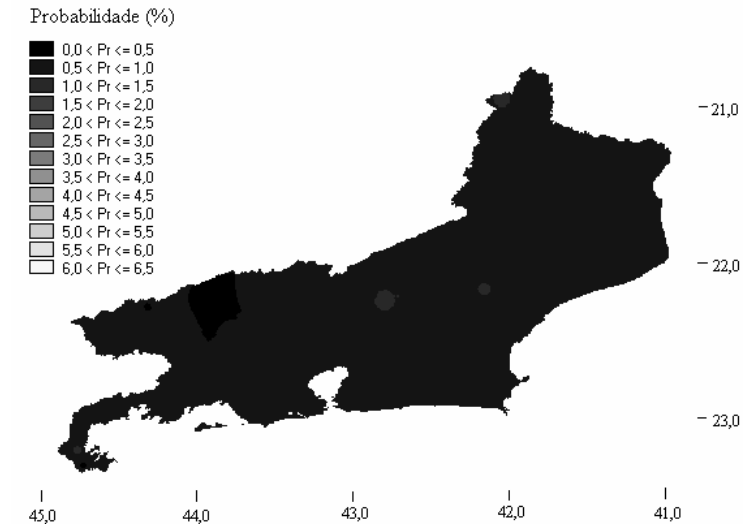

(b)

FIGURA 4. Mapas dos valores de probabilidade de veranico no Estado do Rio de Janeiro com duração de 1 a 5 dias (a), 6 a 10 dias (b), no mês de janeiro.

Para a Figura 5, análises semelhantes podem ser feitas, permanecendo a região Noroeste como sendo aquela onde se observam menores probabilidades de ocorrência de veranicos de pequena duração, contrastando com a região Sul, onde se observam as maiores probabilidades de ocorrência de veranicos de curta duração. Porém, para as demais durações, as probabilidades são bem menores.

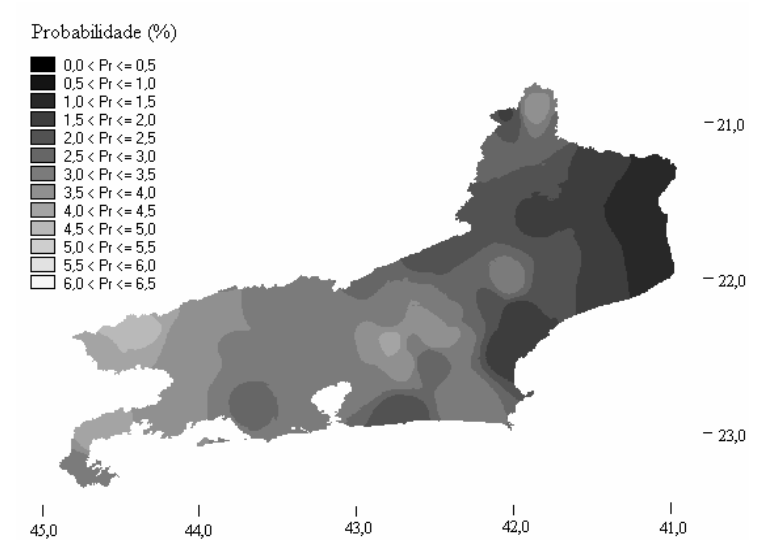

(a)

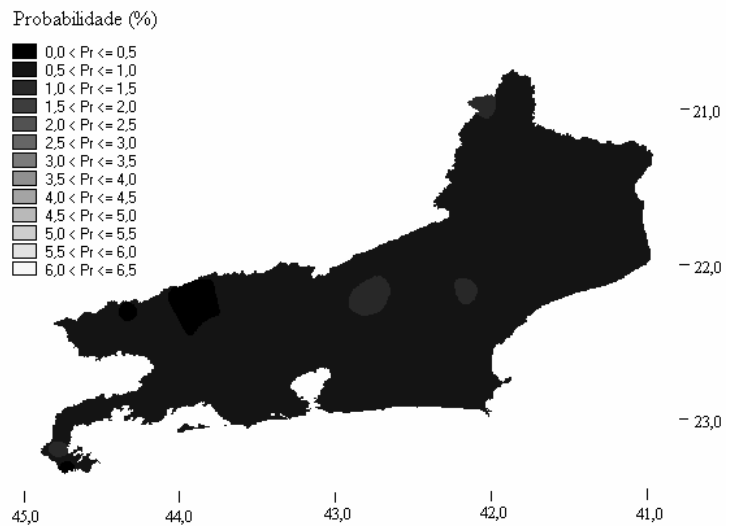

(b)

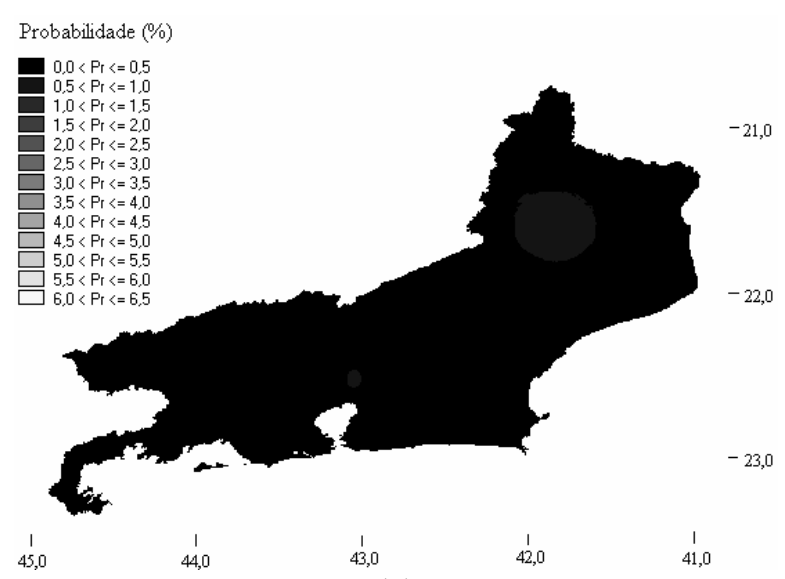

(c)

FIGURA 5. Mapas dos valores de probabilidade de veranico no Estado do Rio de Janeiro com duração de 1-5 dias (a), 6-10 dias (b) e 11-15 dias (c), no mês de fevereiro. 
De acordo com a Tabela 3, que apresenta porcentagens de área do Estado ocupadas com diferentes classes de veranico, no mês de dezembro, 84,2\% da área do Estado apresenta veranicos médios ponderados com duração entre três e cinco dias, não havendo ocorrência desses eventos com durações superiores a sete dias. No mês de janeiro, espera-se comportamento semelhante, sendo que $74,2 \%$ da área do Estado apresenta veranicos médios ponderados com duração entre três e cinco dias, não sendo observados, em média, esses eventos com durações superiores a oito dias. Já para o mês de fevereiro, os veranicos médios ponderados com duração entre três e sete dias podem ser identificados em $84,8 \%$ da área do Estado. Porém, nesse mês, veranicos médios com magnitudes entre dois e onze dias são observados em todo o Estado.

TABELA 3. Porcentagem de área ocupada com intervalos fixos de veranicos médios ponderados, em dias, para dezembro, janeiro e fevereiro, no Estado do Rio de Janeiro.

\begin{tabular}{cccc}
\hline Classes & dezembro & janeiro & fevereiro \\
\hline $2,0<$ ver $\leq 3,0$ & 9,5 & 8,1 & 0,3 \\
$3,0<$ ver $\leq 4,0$ & 71,8 & 52,0 & 17,0 \\
$4,0<$ ver $\leq 5,0$ & 12,4 & 22,3 & 34,1 \\
$5,0<$ ver $\leq 6,0$ & 5,0 & 8,9 & 17,2 \\
$6,0<$ ver $\leq 7,0$ & 1,3 & 7,1 & 16,5 \\
$7,0<$ ver $\leq 8,0$ & --- & 1,6 & 7,1 \\
$8,0<$ ver $\leq 9,0$ & --- & -- & 4,0 \\
$9,0<$ ver $\leq 10,0$ & --- & --- & 2,8 \\
$10,0<$ ver $\leq 11,0$ & --- & -- & 0,9 \\
\hline
\end{tabular}

Os resultados apresentados nas Figuras 3, 4 e 5 e na Tabela 3 são, em parte, explicados por CARVALHO et al. (2003), que desenvolveram um estudo a respeito da precipitação média mensal no Estado, no período de outubro-março. Os autores concluíram que dezembro e janeiro apresentam os mais elevados índices pluviométricos, fazendo com que seqüências maiores de dias secos sejam mais raras de ocorrer nesses meses, principalmente nas regiões Norte e Noroeste do Estado. Os autores concluíram, também, que fevereiro é um mês em que veranicos com diferentes durações são mais fáceis de serem observados quando comparado aos meses de janeiro e dezembro, pois esse é o mês mais seco dentre os três estudados.

\section{Distribuição das classes de probabilidade de ocorrência de veranicos no Estado do Rio de Janeiro}

Na Tabela 4, apresentam-se os valores porcentuais de área do Estado ocupada com as classes de probabilidade utilizadas. Para veranicos com duração de 1 a 5 dias, observa-se que, de dezembro a fevereiro, ocorre uma redução nos valores porcentuais de áreas do Estado ocupadas com as classes de 4,0 a 5,0\% de probabilidade de ocorrência desses eventos. Já para durações de 6 a 10 dias, nos meses de janeiro e fevereiro, uma grande porcentagem de área do Estado está ocupada apenas com a classe de 0,5 a 1,0\% de probabilidade de ocorrência, indicando comportamento uniforme em todo o Estado.

Na Tabela 5, são apresentados os maiores e os menores valores de duração de veranicos médios simulados (valores extremos). Pode-se observar que, em média, fevereiro é o mês que apresenta veranicos com maior duração, a exemplo de SOUSA (1999). Estudando a ocorrência de veranicos no Estado de São Paulo, o autor observou que o mês de maior duração média de veranico foi março, seguido de fevereiro. O mesmo autor apresentou resultados semelhantes com relação ao padrão de distribuição dos veranicos, para os meses de dezembro e janeiro. No presente trabalho, houve comportamento semelhante, sendo que apenas os valores médios máximos tiveram diferentes durações 
para os mesmos meses, porém essas diferenças não são significativas para fins de estudo da ocorrência de veranicos.

TABELA 4. Valores porcentuais de área do Estado ocupada em cada classe de probabilidade de ocorrência de veranicos com durações de 1 a 5 dias, 6 a 10 dias e 11 a 15 dias.

\begin{tabular}{cccccccccc}
\hline \multirow{2}{*}{ Classes } & \multicolumn{3}{c}{ dezembro } & \multicolumn{4}{c}{ janeiro } & \multicolumn{3}{c}{ fevereiro } \\
\cline { 2 - 10 } & 1 a 5 & 6 a 10 & 11 a 15 & 1 a 5 & 6 a 10 & 11 a 15 & 1 a 5 & 6 a 10 & 11 a 15 \\
\hline $0,0<\operatorname{Pr} \leq 0,5$ & --- & 8,5 & 100,0 & --- & 2,5 & 100,0 & -- & 2,5 & 95,3 \\
$0,5<\operatorname{Pr} \leq 1,0$ & --- & 63,4 & --- & --- & $\mathbf{9 6 , 8}$ & --- & --- & $\mathbf{9 4 , 9}$ & 4,7 \\
$1,0<\operatorname{Pr} \leq 1,5$ & --- & 28,2 & --- & --- & 0,7 & --- & 7,2 & 2,6 & --- \\
$1,5<\operatorname{Pr} \leq 2,0$ & 1,1 & --- & --- & 0,5 & --- & --- & 13,5 & --- & --- \\
$2,0<\operatorname{Pr} \leq 2,5$ & 4,8 & --- & --- & 5,3 & --- & --- & 16,0 & --- & --- \\
$2,5<\operatorname{Pr} \leq 3,0$ & 5,1 & --- & --- & 7,2 & --- & --- & 13,6 & --- & --- \\
$3,0<\operatorname{Pr} \leq 3,5$ & 5,9 & --- & --- & 11,4 & --- & --- & $\mathbf{3 0 , 2}$ & --- & --- \\
$3,5<\operatorname{Pr} \leq 4,0$ & 11,9 & --- & --- & 17,5 & --- & --- & 13,5 & --- & --- \\
$4,0<\operatorname{Pr} \leq 4,5$ & $\mathbf{3 3 , 4}$ & --- & --- & $\mathbf{3 0 , 9}$ & --- & --- & 4,4 & --- & --- \\
$4,5<\operatorname{Pr} \leq 5,0$ & $\mathbf{2 5 , 3}$ & --- & --- & 15,2 & --- & --- & 1,6 & --- & --- \\
$5,0<\operatorname{Pr} \leq 5,5$ & 8,4 & --- & --- & 9,3 & --- & --- & --- & --- & --- \\
$5,5<\operatorname{Pr} \leq 6,0$ & 3,2 & --- & --- & 2,7 & --- & --- & --- & --- & --- \\
$6,0<\operatorname{Pr} \leq 6,5$ & 0,7 & --- & --- & 0,1 & --- & --- & --- & --- & --- \\
\hline
\end{tabular}

TABELA 5. Valores extremos de duração de veranicos médios simulados, em dias, para o Estado do Rio de Janeiro, em dezembro, janeiro e fevereiro.

\begin{tabular}{cccc}
\hline Valores & dezembro & janeiro & fevereiro \\
\hline Mínimo & 2,30 & 2,30 & 2,87 \\
Máximo & 6,50 & 7,37 & 10,76 \\
\hline
\end{tabular}

Na Figura 6, evidencia-se a discussão anterior. Nas Figuras 6a e 6b, referentes aos meses de dezembro e janeiro, apresenta-se a distribuição regional dos valores médios ponderados dos veranicos. Observa-se que veranicos com durações inferiores a sete dias predominam em quase todo o Estado, enquanto veranicos com maiores durações são discretamente observados apenas na região Norte. Já na Figura 6c, referente a fevereiro, veranicos com durações maiores que seis dias são observados em quase todo o Estado. Esse fato contribui para um aumento na média dos veranicos observados nesse mês em distinção ao comportamento observado nos dois meses anteriormente citados.

Em linhas gerais, as variações de ocorrência de veranicos observadas neste estudo entre as estações do ano no Centro-Sul do Brasil são dominadas pelos deslocamentos dos limites entre as correntes do Brasil e Malvinas, o que leva a mudanças na temperatura da superfície do mar e na pressão sobre essas massas de água (GARCIA, 1994). Entretanto, as anomalias na distribuição de chuvas sobre o Brasil são parte de um evento mais amplo, em escala global, chamado oscilação do sul ou Southern Oscillation (RAO \& HADA, 1990). Nas oscilações, os eventos de seca são relacionados à formação de uma célula de subsidiência em uma dada região devido ao deslocamento da célula de elevação para outra região (RAO \& HADA, 1990). Eventos mais extremos dessa oscilação do sul são relacionados ao El Niño Southern Oscillation (ENSO), que levam a anomalias mais drásticas na distribuição de chuvas no Brasil (RAO et al., 2001). 


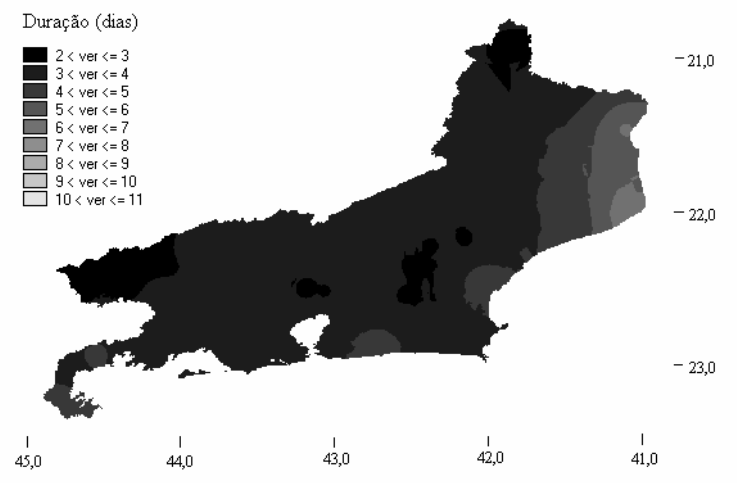

(a)

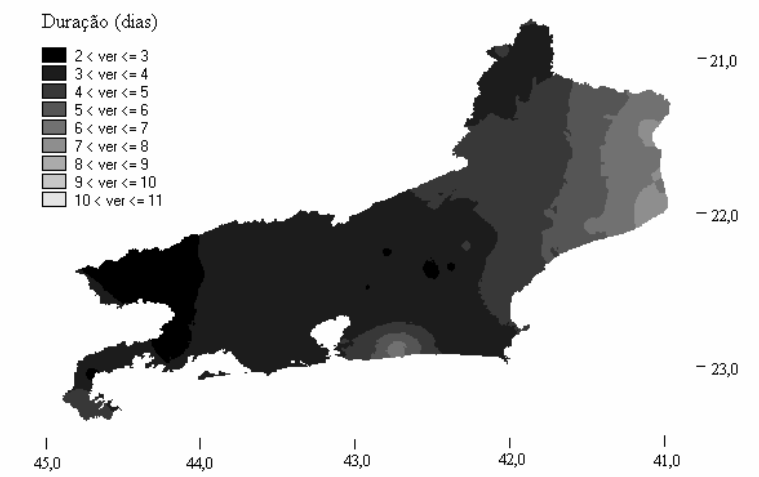

(b)

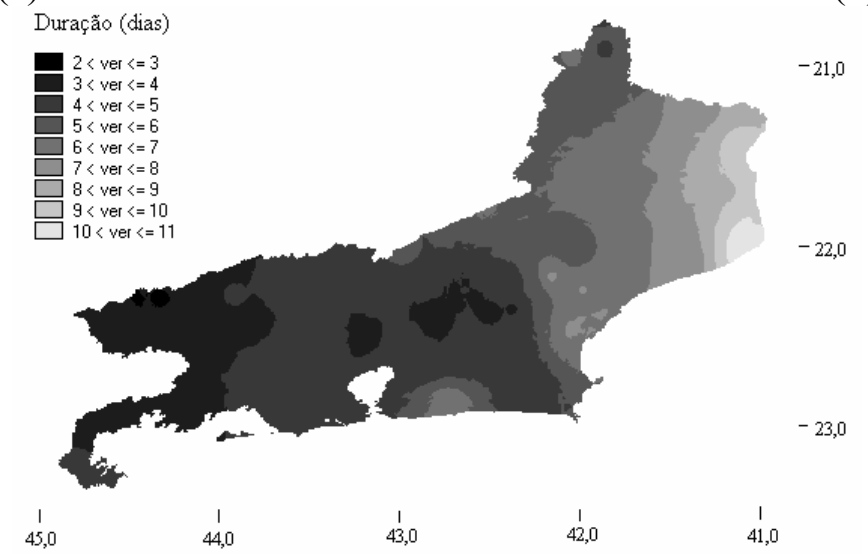

(c)

FIGURA 6. Mapas dos valores médios ponderados de duração do veranico no Estado do Rio de Janeiro para dezembro (a), janeiro (b) e fevereiro (c).

\section{CONCLUSÕES}

A metodologia empregada possibilita a simulação de ocorrência de veranicos com diferentes durações para o Estado do Rio de Janeiro, com alto grau de confiabilidade.

A maior probabilidade de ocorrência de veranicos com 11 a 15 dias de duração se dá no mês de fevereiro, sobretudo na região Norte. duração.

A região Sul apresentou maior probabilidade de ocorrências de veranicos de um a cinco dias de

\section{REFERÊNCIAS BIBLIOGRÁFICAS}

ASSAD, E.D.; SANO, E.E.; BEZERRA, H.S.; SILVA, S.C.; LOBATO, E.J.V. Uso de modelos numéricos de terrenos na espacialização de épocas de plantio. In: ASSAD, E.D.; SANO, E.E. Sistema de informação geográfica - Aplicações na agricultura. 2. ed. Brasília: Embrapa SPI/Embrapa-CPAC, 1998. p.311-69.

CARVALHO, D.F.; CRUZ, E.S.; GUIMARÃES, E.C.; CEDDIA, M.B.; GOMES, B.M. Precipitação média mensal do semestre outubro-março para o Estado do Rio de Janeiro, utilizando métodos geoestatísticos. In: CONGRESSO BRASILEIRO DE ENGENHARIA AGRÍCOLA, 32., 2003, Goiânia. Anais... Goiânia: Sociedade Brasileira de Engenharia Agrícola, 2003. 1 CD.

CARVALHO, D.F.; MARQUES, D.C.; MONTEBELER, C.A.; BARBOSA, L.G.; SANTANA, G.C. Demanda máxima de irrigação para o milho e feijão , no Estado do Rio de Janeiro, Brasil. In: 
CONGRESSO LATINO AMERICANO DE ENGENHARIA AGRÍCOLA, 3., 2000, Guanajuato México. Anais... Guanajuato: Associación Latinoamericana de Ingenieria Agrícola, 2000. 1 CD.

CRISTOFIDIS, D. Recursos hídricos dos cerrados e seu potencial de utilização na agropecuária. In: CONGRESSO BRASILEIRO DE ENGENHARIA AGRÍCOLA, 32., 2003, Goiânia. Anais... Goiânia: Sociedade Brasileira de Engenharia Agrícola, 2003. 1 CD.

GARCIA, N.O. South American Climatology. Quaternary International, St. John's, v.21, p.7-27, 1994.

HASHEMI, F.; DECKER, W. Using climatic information and weather forecast for decisions in economizing irrigation water. Agricultural Meteorology, Amsterdam, v.6, p.245-57, 1969.

NEUMANN, J.; ULAM, S. The monte Carlo method. Journal of the American Statistical Association, Washington, v.44, p.335-41,1949.

PAIVA, C. Determinação das datas de início e fim da estação chuvosa e da ocorrência de veranico, na Bacia do Rio Doce. 1997. 69 f. Dissertação (Mestrado em Meteorologia Agrícola) - Universidade Federal de Viçosa, Viçosa - MG, 1997.

RAO, V.B.; HADA, K. Characteristics of rainfall over Brazil: Annual variations and connections with the Southern Oscillation. Theoretical and Applied Climatology, Wien, v.42, p.81-91, 1990.

RAO, V.B.; SANTO, C. E.; FRANCHITO, S.H. A diagnosis of rainfall over South America during 1997/98 El Niño event. Part I: Validation of NCEP-NCAR reanalysis of rainfall data. Journal of Climate, Boston, v.15, p.502-11. 2001.

SILVA, M.M.P.; ARAÚJO, M.I.M.; ANDRADE, A.M.P.A. Probabilidade de ocorrência de veranicos na baixada campista. Saneamento, Rio de Janeiro, v.51, p.26-33, 1977.

SOBOL, I. O método de Monte Carlo. Trad. De M. DOMBROVSKY. Moscou: Editora Mir Moscou, 1983. $64 \mathrm{p}$.

SOUSA, S.A.V. Programa computacional para simulação da ocorrência de veranicos e queda de produção. 1999. 124 f. Tese (Doutorado em Irrigação e Drenagem) - Escola Superior de Agricultura "Luiz de Queiroz", Universidade de São Paulo, Piracicaba, 1999.

VALERIANO, M.M.; PICINI, A.G.; MELLO, M.H.A. Estratificação de efeitos anisotrópicos do relevo na geração de mapas pluviométricos por geoprocessamento. Revista Brasileira de Agrometeorologia, Santa Maria, v.10, n.1, p.167-77, 2002. 